\title{
Endogenous morphine synthetic pathway preceded and gave rise to catecholamine synthesis in evolution (Review)
}

\author{
GEORGE B. STEFANO ${ }^{1}$ and RICHARD M. KREAM ${ }^{2}$ \\ ${ }^{1}$ Neuroscience Research Institute, State University of New York - College at Old Westbury, Old Westbury, NY; \\ ${ }^{2}$ Department of Biochemistry, Downstate Medical Center, State University of New York, Brooklyn, NY, USA
}

Received May 2, 2007; Accepted June 11, 2007

\begin{abstract}
The biological presence and regulatory function of the plant alkaloid morphine in relatively simple and complex integrated animal systems has previously been shown. The pivotal role of dopamine as a chemical intermediate in the morphine biosynthetic pathway in plants establishes a functional basis for its expansion into an essential role as the progenitor catecholamine signaling molecule. In invertebrate neural systems, dopamine serves as the preeminent catecholamine signaling molecule, with the emergence and limited utilization of norepinephrine and its biosynthetic enzyme dopamine $\beta$-hydroxylase in newly defined adaptational chemical circuits required by a rapidly expanding set of physiological demands. In vertebrates, epinephrine emerges as the major end of the catecholamine synthetic pathway consistent with a newly incorporated regulatory modification, i.e. N-methylation of norepinephrine. Given the striking similarities between the enzymatic steps in the morphine biosynthetic pathway and those driving the evolutionary adaptation of catecholamine chemical species to accommodate an expansion of interactive but distinct signaling systems, we surmise that the evolutionary emergence of catecholamine systems required conservation and selective 'retrofit' of specific enzyme activities, i.e. catechol O-methyl transferase and phenylethanol-amine $\mathrm{N}$-methyl transferase, drawn from cellular morphine expression. This hypothesis is further supported by the critical recruitment of enzymatically synthesized tetrahydrobiopterin (BH4) both as an essential cofactor for tyrosine hydroxylase-mediated dopamine production and as a secondary electron donor for nitric oxide synthase-mediated nitric oxide (NO) production. The establishment of a reciprocal regulatory linkage between NO
\end{abstract}

Correspondence to: Dr George B. Stefano, Neuroscience Research Institute, SUNY College at Old Westbury, P.O. Box 210, Old Westbury, NY 11568 , USA

E-mail: gstefano@sunynri.org

Key words: morphine, mu3 opiate receptor subtype, catecholamine, dopamine, tetrahydrobiopterin, guanosine triphosphate cyclohydrolase and catecholaminergic processes, as mediated by $\mathrm{BH} 4$, subserves a pivotal capacity to promote autocrine and paracrine regulation of signaling molecules. In summary, ongoing development and adaptation of catecholamine signaling pathways in animals appear to be related to their mobile lifestyle associated with complex feeding, sexual and protective processes, which also generate free radicals, thus requiring morphinergic signaling coupling to NO release.

\section{Contents}

1. Introduction

2. Enzyme evolvement

3. Intracellular signaling

4. $\mu 3$ : Supporting the morphinergic presence

\section{Introduction}

In examining the literature regarding morphine biosynthesis, it is common knowledge that plants can make morphine (1-15). In this same literature, one can find evidence for components of the catecholamine pathway in plants, yet they do not, as best as we can determine, make or use these monoamines as signaling agents. In invertebrates, it has recently been demonstrated that the ability to make morphine is present in a manner similar to that found in plants (16-18). However, unlike plants, catecholamine signaling emerges in invertebrates with dopamine as the major molecule used in neural systems (19-21). There are even a few reports in long-lived invertebrates that norepinephrine is present, however, always at low levels (22). Importantly, despite numerous attempts, our laboratory could not identify epinephrine in invertebrate or plant tissues, suggesting that this synthesis had not emerged hundreds of millions of years ago. In vertebrates, it is widely known that the catecholamine pathway is present and it terminates in the synthesis of epinephrine (23). Recently, in healthy and normal human tissues we demonstrated that morphine can be made (24). Taken together, both plants and animals make morphine, and only in vertebrates is the catecholamine pathway complete, strongly suggesting that catecholamine biosynthesis emerged from the morphine biosynthetic pathway since both depend on tyrosine, L-DOPA, dopamine (DA) and tyramine $(18,24,25)$ (Fig. 1). 


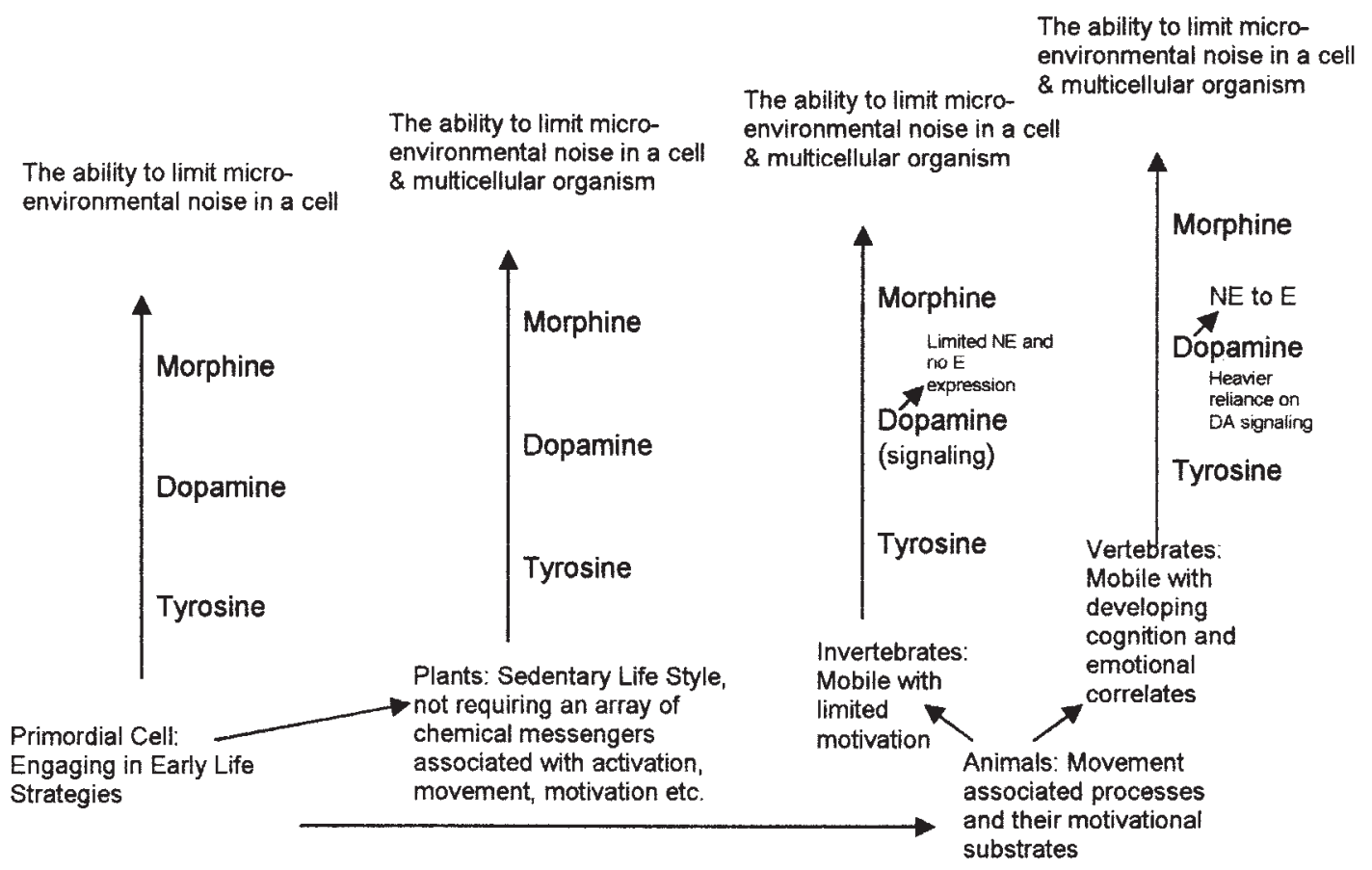

Figure 1. Requirements - evolutionary pressure. Primordial and plant cells do not use catecholamine signaling, even though dopamine may be present. Invertebrates use DA as a signaling molecule and in certain long-lived invertebrates the hydroxylase is modified to make dopamine $\beta$-hydroxylase. In vertebrates, catechol O-methyl transferase (COMT) from the morphine pathway is modified to accommodate norepinephrine (NE) to form epinephrine (E). Furthermore, highlighting this relationship even further is an association of catecholamine brain localization with that of endogenous morphine $(16,18,29,59-65)$. This association may be predicted from the fact that DA is an endogenous morphine precursor, and the ability of morphine-induced NO to downregulate excitatory processes and scavenge free radicals, in part, is associated with catecholamine signaling $(28,42)$.

Speculating further, the question arises as to what pressure or need forced this new signaling pathway, i.e. catecholamine, to emerge in its own right? We surmise, given numerous reports from many laboratories, that morphine exerts general downregulation of tissue excitability via highly specific cellular and receptor-mediated processes, protecting tissues from over excitability (26-29). This is also true for its actions in plants (3). Therefore, with the advance of more complex motor activities in invertebrates and vertebrates associated with complex feeding, sexual and protective processes, a new signaling system had to emerge, namely, in the form of DA in invertebrates. Thus, DA may serve as a major signaling molecule associated with a mobile lifestyle found in these animals.

It is our contention that this dopaminergic regulatory function associated with mobility was so successful in invertebrates that it was continued in vertebrates with further amplification to fully establish norepinephrine and epinephrine signaling, further dividing the motor activation processes to better suit a more sophisticated mobile lifestyle. Thus, the initial motor-associated activating system and its associated behaviors were amplified not only to include new complex motor activities, but motivation processes, i.e. reward, pleasure and pain associated with the use of these systems, was also expanded and developed. Hence, we have the well-recognized links between motor activities and emotional neural processes. We further surmise that with the advent of the catecholamine processes, including emotion, cognition emerged as a coping strategy serving as yet another means to activate motor processes in a more focused manner, providing for higher survival strategies $(30,31)$.

\section{Enzyme evolvement}

Positive evolutionary pressure mediates the differentiation of relatively simple cellular systems into complex organ systems. The novel hypothesis presented above must also embrace the evolutionary role of endogenous morphine as a prototype molecular principle by which the exponential increases in cellular differentiation required for adaptation and utilization of the related family of catecholamines as signaling molecules are modeled. In support of our contention, the emergence of catecholaminergic signaling systems was facilitated by the genetic 'retrofit' of a common set of enzymes within the morphine biosynthetic pathway to accommodate biochemical maturation and modification of DA-related compounds (25). Notably, the plant N-methyl and O-methyl transferases, required for conversion of the essential morphine precursor norlaudanosoline (also called tetrahydropapaveroline, THP) to the pre-morphinan alkaloid S-reticuline, have been adaptively transformed into major enzymes in catecholamine expression, i.e. phenylethanolamine N-methyl transferase (PNMT) and catechol O-methyl transferase (COMT), respectively. Accordingly, the incremental evolutionary adaptations of DA necessary for the cellular expression and utilization of epinephrine as a neural/ neuroendocrine signaling molecule required co-ordinated recruitment and complex regulation of PNMT within tyrosine hydroxylase (TH)- and dopamine $\beta$-hydroxylase (DBH)positive cells (22).

Based on this novel hypothesis that catecholamines emerged from the conserved morphine pathway is the fact that COMT has always been a part of the morphine pathway 


\section{Normal Expression}

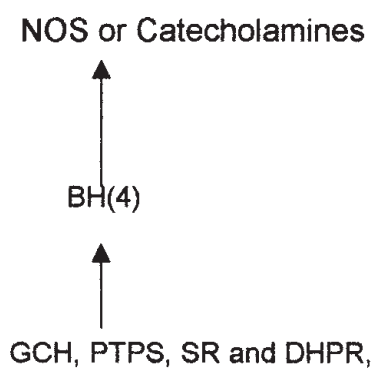

Acute Morphine Exposure Significantly lowers their levels

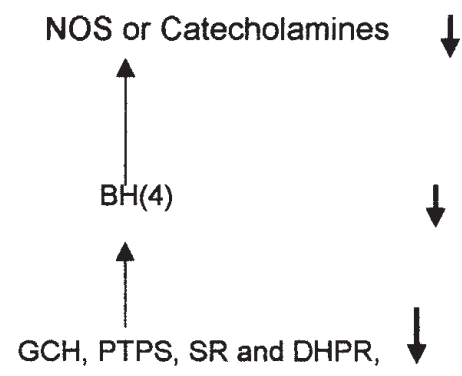

Figure 2. Demonstrating the ability of morphine as an autocrine and paracrine regulator, acute morphine exposure downregulates both factors its synthesis depends on or via end production inhibition as is the case for dopamine or nitric oxide (49). DHPR, dihydropteridine reductase; PTPS, 6-pyruvoyltetrahydropterin synthase; SR, sepiapterin reductase; NOS, nitric oxide synthase; GCH, guanosine triphosphate cyclohydrolase; $\mathrm{BH}(4)$, tetrahydrobiopterin.

(25), yet epinephrine is not present in plants and not expressed by invertebrate tissues. This also supports catecholamine emergence from the morphine pathway hypothesis, since elapsed time had to occur along with a functional need to make yet another signal molecule. Thus, in order to further develop the catecholamine pathway, time had to elapse so that COMT/PNMT would undergo a transformation to accommodate the conversion of norepinephrine into epinephrine. In this regard, tyrosine hydroxylase preceded DBH in the evolutionary scheme, reflecting the appearance of norepinephrine in select long-lived invertebrates that required a higher level of motor-associated mobilization strategies (22).

\section{Intracellular signaling}

To accommodate the 'newly arrived' catecholamine pathway, intracellular signal molecules also had to be 'redesigned/ stereospecifically matched' simply to assure conformational matching so that the highly specific stereoselect nature of their regulation was possible. In this regard we are drawn to nitric oxide (NO) signaling because of its uniform presence in living organisms and its coupling to morphine signaling (28,32-34). Initially, it was demonstrated that vascular dilation is mediated to a large extent by endothelium-dependent NO, produced by the enzyme nitric oxide synthase (NOS) in the presence of the cofactor tetrahydrobiopterin ( $\mathrm{BH} 4)$, found only in animals, as well as mediated via the cGMP-dependent downstream signaling cascade (35-39), found in both plants and animals. Thus, levels of BH4 in complex animal cellular systems determine if NO becomes uncoupled from arginine oxidation, and NOS produces superoxide rather than NO in animals. Clearly, this uncoupling may represent an important component in various disorders associated with oxidative stress and free radical generation in diverse tissues $(28,40-44)$.

Upon re-examining the literature, we found that GTP cyclohydrolase $(\mathrm{GCH})$ appears to be the rate-limiting enzyme for BH4 synthesis in animals, and thus has been shown to be a key modulator of peripheral neuropathic and inflammatory pain, which is also linked to NO signaling $(34,45)$. Importantly, $\mathrm{BH} 4$ is also an essential cofactor for catecholamine production in animals, i.e. TH, enhancing its significance further especially since this pathway extends from morphine biosynthesis in animals.

It is important to note that plants do not require $\mathrm{BH} 4$ since they use $\mathrm{GCH}$, which also represents the first step in the de novo tetrahydrofolate biosynthetic pathway present in bacteria, fungi, and plants, and encoded in Escherichia coli by the folE gene (46). It is also the first enzyme of the biopterin (BH4) pathway in Homo sapiens, where it is encoded by a homologous folE gene (46). Thus, plants require only GCH, most likely because DA is not used as a signal molecule just an intermediate in morphine biosynthesis. With the advent of additional signal molecules, i.e. catecholamines, we surmise that $\mathrm{BH} 4$ also evolved/adapted to limit or regulate excessive reactive molecules, such as DA and its by-products, from emerging and initiating tissue damage as free radicals. This same logic was employed to limit NOS activity, given the enhanced free radical potential via catecholamine biosynthesis. There is a dynamic relationship between $\mathrm{BH} 4$ bound to $\mathrm{TH}$ and free $\mathrm{BH} 4$ that stimulates DOPA release, suggesting this association is important in morphine synthesis since DOPA serves as a morphine precursor $(25,47)$. Supporting a dynamic role for BH4, not only is it a cofactor for $\mathrm{TH}$ but a direct regulator of intracellular concentration of TH (48). Thus, its use by the catecholamine pathway separates its general regulatory sphere of influence from that of $\mathrm{GCH}$ in that it performs activities associated with NOS and TH modulation, which is reciprocal in nature (49). This relationship is even more important in that $\mathrm{GCH}$ controls the biosynthesis of $\mathrm{BH} 4$, indicating that it also preceded the role of BH4 in animal cells. From this we can surmise that BH4 evolvement occurred because it had the stereospecific ability to modulate NOS and TH actions.

Supporting this hypothesis further is the fact that NOS exhibits similarities with CYP 450 (50). Both are hemethiolate proteins, employing the same prosthetic group to perform similar chemistry. They also share the same redox partner, a diflavoprotein reductase, which in the case of NOS is incorporated with the oxygenase in one polypeptide chain. The major difference is the presence in NOS of the additional cofactor tetrahydrobiopterin, which is applied as an auxiliary electron donor to prevent decay of the oxyferrous complex to ferric heme and superoxide, which manages the balance 
between free radical generation and NO. Thus, CYP 450 variants and $\mathrm{GCH}$ could not serve as an adequate substitute for $\mathrm{BH} 4$, which is specifically designed to modulate this pathway given its unique properties with the advent of catecholamines in animal evolution as a spin-off from morphine biosynthesis.

Additionally, it has been shown that in a DA cell, which is NOS positive, NO has significant and positive effects on cell survival that are anti-apoptotic (51). Recent critical studies have reported the association between NO produced by a specific isotype found in the mitochondrion, i.e. mitochondrial NOS (mtNOS), in regulating cellular oxygen consumption/ energy metabolism without engendering oxidative stress $(52,53)$. Interestingly, an association has been made between mtNOS and a NOS enzyme species found in plants (54), demonstrating that plant components are still found in animal cells apparently modulating intracellular actions.

Taken together, our recent elucidation of a de novo morphine biosynthetic pathway in animal cells, with strikingly similar characteristics to that found in opium poppy, and the ability of morphine to stimulate NOS-derived NO release, strongly suggests that evolutionary pressure has conserved primordial regulatory circuitry (25). It also suggests that this system has been carried over from plants and manifests itself in energy and developmental processes (55).

\section{4. $\mu 3$ : Supporting the morphinergic presence}

In 1993, pharmacological evidence was presented for the presence of the $\mu 3$ opiate receptor subtype, which is opioid peptide insensitive and morphine selective, on human and invertebrate tissues (56). Ten years later it was cloned on human immune, vascular and neural tissues and was found to be a mu splice variant (57). During this period, it was also demonstrated that this receptor was coupled to constitutive NO production in these diverse human tissues and those of other animals as well, including invertebrates $(27-29,32,57)$. We surmised that the general yet specific cellular downregulatory processes of morphine were mediated by constitutive NO (28). The ability of $\mu 3$ to also gate intracellular calcium transients, eventually affecting mitochondrial oxygen consumption and energy conservation, provides a compelling functional linkage of this receptor with recently characterized calcium channels (58). Thus, these evolutionarily conserved signaling and regulatory processes were coupled and functionally linked.

The significance of the $\mu 3$ opiate receptor is further enhanced by our recent finding that it is present on human stem cells not induced to differentiate (unpublished), which did not contain other types of mu receptors. Taken together, in all probability the $\mu 3$ morphine receptor was probably the first in the line of future opioid receptor types that evolved in animals.

With the discovery of the $\mu 3$ opiate receptor and its coupling to constitutive NO release came the mechanism to 'manage' the evolvement of catecholamine signaling, which was needed to effectively control catecholamine activationtype processes (feeding, movement, motivation, sex). Fig. 2 dramatically illustrates the downregulatory action of morphine on the BH4 pathway. Taken together, out of the 'calming' homeostatic processes there later emerged a system involved with selective activation (e.g. catecholamine), which would then be downregulated when their goal was accomplished.

In conclusion, it comes as no surprise to comprehend what happens when variations/errors creep into the modulation of the activation processes (e.g. BH4, GCH, COMT/PNMT). Furthermore, the catecholamine emergence from the older morphinergic biosynthesis pathway provides a compelling rationale for the coupling of literature of these and other ancillary pathways into morphinergic signaling processes, providing the missing knowledge for various disciplines such as substance abuse, as well as mental health, since both depend on a catecholamine signaling substrate.

\section{Acknowledgements}

This study was supported in part by grants MH 47392 and DA 09010 and the Empire Innovation Program of New York State.

\section{References}

1. Bird DA and Facchini PJ: Berberine bridge enzyme, a key branch-point enzyme in benzylisoquinoline alkaloid biosynthesis, contains a vacuolar sorting determinant. Planta 213: 888-897, 2001.

2. Facchini PJ and De Luca V: Differential and tissue-specific expression of a gene family for tyrosine/dopa decarboxylase in opium poppy. J Biol Chem 269: 26684-26690, 1994.

3. Facchini PJ and Park SU: Developmental and inducible accumulation of gene transcripts involved in alkaloid biosynthesis in opium poppy. Phytochemistry 64: 177-186, 2003.

4. Facchini PJ and De Luca V: Expression in Escherichia coli and partial characterization of two tyrosine/dopa decarboxylases from opium poppy. Phytochemistry 38: 1119-1126, 1995.

5. Park SU, Johnson AG, Penzes-Yost C and Facchini PJ: Analysis of promoters from tyrosine/dihydroxyphenylalanine decarboxylase and berberine bridge enzyme genes involved in benzylisoquinoline alkaloid biosynthesis in opium poppy. Plant Mol Biol 40: 121-131, 1999.

6. Samanani $\mathrm{N}$ and Facchini PJ: Isolation and partial characterization of norcoclaurine synthase, the first committed step in benzylisoquinoline biosynthesis, from poppy. Planta 213: 898-906, 2001

7. Samanani N and Facchini PJ: Purification and characterization of norcoclaurine synthase. The first committed enzyme in benzylisoquinoline alkaloid biosynthesis in plants. J Biol Chem 277: 33878-33883, 2002.

8. De-Eknamkul W and Zenk MH: Enzymatic formation of (R)reticuline from 1,2-dehydroreticuline in the opium poppy plant. Tetrahedron Lett 31: 4855-4858, 1990.

9. Frenzel T and Zenk MH: S-Adenosyl-L-methionine: 3'hydroxy- $N$-methyl- $(S)$-coclaurine 4 '- $O$-methyltransferase, a regio- and stereoselective enzyme of the $(S)$-reticuline pathway. Phytochemistry 29: 3505-3511, 1990.

10. Gerady R and Zenk MH: Formation of salutaridine from (R)reticuline by a membrane-bound cytochrome $\mathrm{P}-450$ enzyme from Papaver somniferum. Phytochemistry 32: 79-86, 1992.

11. Herbert RB: The biosynthesis of isoquinoline alkaloids. In: The Chemistry and Biology of Isoquinoline Alkaloids. Phillipson JD, Roberts MF and Zenk MH (eds). Springer-Verlag, Berlin, Heidelberg pp213-228, 1985.

12. Lenz $\mathrm{R}$ and Zenk MH: Closure of the oxide bridge in morphine biosynthesis. Tetrahedron Lett 35: 3897-3900, 1994.

13. Lenz R and Zenk MH: Stereospecific reduction of codeinone, the penultimate enzymatic step during morphine biosynthesis in Papaver somniferum. Tetrahedron Lett 36: 2449-2452, 1995.

14. Lenz $\mathrm{R}$ and Zenk MH: Purification and properties of codeinone reductase (NADPH) from Papaver somniferum cell cultures. Eur J Biochem 233: 132-139, 1995.

15. Loeffler S and Zenk MH: The hydroxylation step in the biosynthesis pathway leading from norcoclaurine to reticuline. Phytochemistry 29: 3499-3503, 1990. 
16. Zhu W, Ma Y, Bell A, et al: Presence of morphine in rat amygdala: Evidence for the mu3 opiate receptor subtype via nitric oxide release in limbic structures. Med Sci Monit 10: BR433-BR439, 2004.

17. Zhu W, Mantione KJ, Shen L and Stefano GB: In vivo and in vitro L-DOPA exposure increases ganglionic morphine levels. Med Sci Monit 11: MS1-MS5, 2005.

18. Zhu W, Mantione KJ, Shen L, et al: Tyrosine and tyramine increase endogenous ganglionic morphine and dopamine levels in vitro and in vivo: CYP2D6 and tyrosine hydroxylase modulation demonstrates a dopamine coupling. Med Sci Monit 11: BR397-BR404, 2005.

19. Stefano GB and Aiello E: Histoflourescent localization of serotonin and dopamine in the nervous system and gill of Mytilus edulis (Bivalvia). Biol Bull 148: 141-156, 1975.

20. Stefano GB, Catapane EJ and Aiello E: Dopaminergic agents: Influence on serotonin in the molluscan nervous system. Science 194: 539-541, 1976.

21. Stefano GB: Comparative aspects of opioid-dopamine interaction. Cell Mol Neurobiol 2: 167-178, 1982.

22. Stefano GB: Norepinephrine: Presence and interaction with endogenous biogenic amines. In: Neurobiology of Mytilus Edulis. Stefano GB (ed). Manchester University Press, Manchester pp93-103, 1990.

23. Iversen LL, Iversen SD and Snyder SH: Biochemistry of Biogenic Amines. Plenum Press, New York, 1975.

24. Zhu W, Cadet P, Baggerman G, Mantione KJ and Stefano GB: Human white blood cells synthesize morphine: CYP2D6 modulation. J Immunol 175: 7357-7362, 2005.

25. Kream RM and Stefano GB: De novo biosynthesis of morphine in animal cells: An evidence-based model. Med Sci Monit 12: RA207-RA219, 2006.

26. Stefano GB and Scharrer B: Endogenous morphine and related opiates, a new class of chemical messengers. Adv Neuroimmunol 4: 57-68, 1994.

27. Stefano GB: Autoimmunovascular regulation: Morphine and anandamide stimulated nitric oxide release. J Neuroimmunol 83: 70-76, 1998 .

28. Stefano GB, Goumon Y, Bilfinger TV, Welters I and Cadet P: Basal nitric oxide limits immune, nervous and cardiovascular excitation: Human endothelia express a mu opiate receptor. Prog Neurobiol 60: 513-530, 2000.

29. Stefano GB, Goumon Y, Casares F, et al: Endogenous morphine. Trends Neurosci 9: 436-442, 2000.

30. Stefano GB and Fricchione GL: The biology of deception: The evolution of cognitive coping as a denial-like process. Med Hypotheses 44: 311-314, 1995.

31. Stefano GB and Fricchione GL: The biology of deception: Emotion and morphine. Med Hypotheses 49: 51-54, 1995.

32. Stefano GB, Scharrer B, Smith EM, et al: Opioid and opiate immunoregulatory processes. Crit Rev Immunol 16: 109-144, 1996.

33. Stefano GB and Liu Y: Opiate antagonism of opioid actions on immunocyte activation and nitric oxide release. Anim Biol 1: 11-16, 1996.

34. Pryor SC, Zhu W, Cadet P, Bianchi E, Guarna M and Stefano GB: Endogenous morphine: opening new doors for the treatment of pain and addiction. Expert Opin Biol Ther 5: 893-906, 2005.

35. Moncada S, Higgs A and Furchgott R: International Union of Pharmacology Nomenclature in Nitric Oxide Research. Pharmacol Rev 49: 137-142, 1997.

36. Ignarro LJ: Signal transduction mechanisms involving nitric oxide. Biochemical Pharmacology 41: 485-490, 1991.

37. Moncada S, Palmer RMJ and Higgs EA: Nitric oxide: physiology, pathophysiology, and pharmacology. Pharmacol Rev 43: 109-142, 1991.

38. Moncada S and Higgs A: The L-arginine-nitric oxide pathway. New Eng J Med 329: 2002-2012, 1993.

39. Moncada S: Nitric oxide in the vasculature: physiology and pathophysiology. Ann NY Acad Sci 811: 60-67, 1997.

40. Fimiani C, Liberty T, Aquirre AJ, Amin I, Ali N and Stefano GB: Opiate, cannabinoid, and eicosanoid signaling converges on common intracellular pathways: Nitric oxide coupling. Prostaglandins Lipid Mediators 57: 23-34, 1999.

41. Welters ID, Fimiani C, Bilfinger TV and Stefano GB: NF-kB, nitric oxide and opiate signaling. Med Hypotheses 54: 263-268, 1999.

42. Benz D, Cadet P, Mantione K, Zhu W and Stefano GB: Tonal nitric oxide and health: A free radical and a scavenger of free radicals. Med Sci Monit 8: 1-4, 2002.
43. Esch T, Stefano GB, Fricchione GL and Benson H: Stressrelated diseases: A potential role for nitric oxide. Med Sci Monit 8: RA103-RA118, 2002.

44. Stefano GB and Esch T: Integrative medical therapy: Examination of meditation's therapeutic and global medicinal outcomes via nitric oxide (Review). Int J Mol Med 16: 621-630, 2005.

45. Tegeder I, Costigan M, Griffin RS, et al: GTP cyclohydrolase and tetrahydrobiopterin regulate pain sensitivity and persistence. Nat Med 12: 1269-1277, 2006.

46. E1-Yacoubi B, Bonnett S, Anderson JN, Swairjo MA, Iwata-Reuyl D and de Crecy-Lagard V: Discovery of a new prokaryotic type I GTP cyclohydrolase family. J Biol Chem 281: 37586-37593, 2006.

47. Breu-Gonzalez P, Gonzalez-Hernandez T, Fonso-Oramas D, Cruz-Muros I, Barroso-Chinea P and Gonzalez MC: Tetrahydrobiopterin stimulates L-DOPA release from striatal tissue. Eur J Pharmacol 541: 33-37, 2006.

48. Urano F, Hayashi N, Arisaka F, Kurita H, Murata S and Ichinose H: Molecular mechanism for pterin-mediated inactivation of tyrosine hydroxylase: formation of insoluble aggregates of tyrosine hydroxylase. J Biochem 139: 625-635, 2006

49. Werner-Felmayer G, Golderer G and Werner ER: Tetrahydrobiopterin biosynthesis, utilization and pharmacological effects. Curr Drug Metab 3: 159-173, 2002.

50. Gorren AC and Mayer B: Nitric-oxide synthase: A cytochrome P450 family foster child. Biochim Biophys Acta 1770: 432-445, 2007.

51. Xu Q, Wink DA and Colton CA: Nitric oxide production and regulation of neuronal NOS in tyrosine hydroxylase containing neurons. Exp Neurol 188: 341-350, 2004.

52. Giulivi $\mathrm{C}$, Kato $\mathrm{K}$ and Cooper $\mathrm{CE}$ : Nitric oxide regulation of mitochondrial oxygen consumption I: cellular physiology. Am J Physiol Cell Physiol 291: C1225-C1231, 2006.

53. Kato $\mathrm{K}$ and Giulivi C: Critical overview of mitochondrial nitricoxide synthase. Front Biosci 11: 2725-2738, 2006.

54. Guo FQ, Okamoto $M$ and Crawford NM: Identification of a plant nitric oxide synthase gene involved in hormonal signaling. Science 302: 100-103, 2003.

55. Riobo NA, Melani M, Sanjuan N, et al: The modulation of mitochondrial nitric-oxide synthase activity in rat brain development. J Biol Chem 277: 42447-42455, 2002.

56. Stefano GB, Digenis A, Spector S, et al: Opiate-like substances in an invertebrate, an opiate receptor on invertebrate and human immunocytes, and a role in immunosuppression. Proc Natl Acad Sci USA 90: 11099-11103, 1993.

57. Cadet P, Mantione KJ and Stefano GB: Molecular identification and functional expression of mu3, a novel alternatively spliced variant of the human mu opiate receptor gene. J Immunol 170: 5118-5123, 2003.

58. Kotturi MF, Hunt SV and Jefferies WA: Roles of CRAC and Cav-like channels in T cells: more than one gatekeeper? Trends Pharmacol Sci 27: 360-367, 2006.

59. Bianchi E, Alessandrini C, Guarna $M$ and Tagliamonte A: Endogenous codeine and morphine are stored in specific brain neurons. Brain Res 627: 210-215, 1993.

60. Bianchi E, Guarna M and Tagliamonte A: Immunocytochemical localization of endogenous codeine and morphine. Adv Neuroimmunol 4: 83-92, 1994.

61. Esch T, Guarna M, Bianchi E, Zhu W and Stefano GB: Commonalities in the central nervous system's involvement with complementary medical therapies: Limbic morphinergic processes. Med Sci Monit 10: MS6-MS17, 2004.

62. Guarna M, Bianchi E, Bartolini A, et al: Endogenous morphine modulates acute thermonociception in mice. J Neurochem 80: 271-277, 2002.

63. Guarna M, Ghelardini C, Galeotti N, Stefano GB and Bianchi E: Neurotransmitter role of endogenous morphine in CNS. Med Sci Monit 11: RA190-RA193, 2005.

64. Neri C, Guarna M, Bianchi E, Sonetti D, Matteucci G and Stefano GB: Endogenous morphine and codeine in the brain of non-human primate. Med Sci Monit 10: MS1-MS5, 2004.

65. Zhu W, Ma Y, Cadet P, et al: Presence of reticuline in rat brain: A pathway for morphine biosynthesis. Mol Brain Res 117: 83-90, 2003. 